\title{
Non-redundant and Natural Variables Definition of Heat Valid for Open Systems
}

\author{
Juan Ramón González Álvarez * \\ C:/Simancas 26, E-36208 (Bouzas) Vigo, Pontevedra, Spain \\ E-mail: oficina@juanrga.com
}

\begin{abstract}
Although an unambiguous definition of heat is available for closed systems, the question of how best to define heat in open systems is not yet settled. After introducing a set of physical requirements for the definition of heat, this article reviews the non-equivalent definitions of heat for open systems used by various authors, emphasizing which physical requirements are not met. A subsequent section deals with the main objective of this article and introduces a new definition of heat that avoids the difficulties of the existent definitions, providing (i) a complete distinction between open and closed systems, (ii) non-redundancy, (iii) natural variables for the thermodynamic potentials, and (iv) a sound, complete, and intuitive generalization of classical thermodynamic expressions.
\end{abstract}

Keywords: First law;second law; heat; open systems.

\section{Introduction}

The true nature of heat, as a form of energy that can interconvert to other forms of energy, was established after much debate in the last part of the $19^{\text {th }}$ century [1] However, and unambiguous definition of heat had been lacking until G. H. Bryan introduced his definition in 1907 $[2]^{\mathbf{a}}$

$\delta Q \equiv d U-\delta W_{T D}$

with $U$ and $W_{T D}$ being the internal energy and thermodynamic work, respectively. Fourteen years later, Born generalized this definition [3] when he considered variations in the total energy $E$ and total work $W$

$\delta Q \equiv d E-\delta W$

The Bryan \& Born definition Eq. (2) is not valid for open systems [1,3]; i.e., for systems that can interchange matter as well as energy. This restriction was not a difficulty in the past, because the basic problem of classical thermodynamics is "the determination of the equilibrium state that eventually results after the removal of internal constraints in [an isolated], composite system" [4]. ${ }^{\mathbf{b}}$

The difficulties began with the extension of classical thermodynamics to open systems, but the problem of defining heat flux becomes more compelling and acute in irreversible thermodynamics. In modern thermodynamics [1], systems in a nonequilibrium thermodynamics state are divided into small elements of volume and each element is assumed to be locally at equilibrium. ${ }^{\mathbf{c}}$ Now, these elements of volume can interchange matter with adjacent elements, which requires a definition of heat valid for open systems as well.

The importance of a generalization of the closedsystems definition of heat has been emphasized many years ago; however, despite the existence of some proposals [5], the question of how best to define heat transfer in open systems is not still settled.
The next section gives an introduction to the more basic formalism of modern thermodynamics, including balance equations for general thermodynamic quantities, and introduces a set of physical requirements for the definition of heat. Section 3 reviews the available non-equivalent definitions of heat for simple thermodynamic open systems, emphasizing what physical requirements are not met.

Section 4 presents a new definition of heat that avoids the main difficulties of the existent definitions. In Section 5, the new definition is introduced for general thermodynamic systems.

\section{Physical Requirements for the Definition of Heat}

Consider a general thermodynamic quantity $Y$, whose density is denoted by $y$. The usual local form of the balance equation for this quantity is [1]

$\frac{\partial y}{\partial t}=\sigma_{Y}-\nabla \cdot \mathbf{J}_{Y}$

where $t$ is time, $\nabla$ is the nabla operator, $\mathbf{J}_{Y}$ is the local flux ${ }^{\mathbf{d}}$ of $Y$, and $\sigma_{Y}$ the amount of $Y$ produced per unit volume and per unit time. Specific expressions for $\sigma_{Y}$ for several quantities such as internal energy, amount of substance, entropy and others are given in the literature [1].

The change in the amount of $Y$ in a volume $V$ in a time $d t$ can be obtained by integrating Eq. (3) over the whole volume, multiplying both sides by $d t$, and applying Gauss' theorem [3]

$d Y=d_{i} Y+d_{e} Y$

in which $d_{e} Y \equiv-d t \int_{A} \mathbf{J}_{Y} \cdot d \mathbf{A}$-with $d \mathbf{A}$ the vector representing an area element- is the change in $Y$ exclusively due to exchanges with the exterior, whereas $d_{i} Y \equiv d t \int_{V} \sigma_{Y} d V$ is the change produced by processes in the interior of the thermodynamic system.

Finally, it must be emphasized that modern thermodynamics does not rely on the use of imperfect 
differentials, because modern thermodynamics is defined over an extended thermodynamic space that includes time. ${ }^{\mathrm{e}}$ This means that classical thermodynamics expressions such as Eq. (2) are modernized to

$d Q \equiv d E-d W$

With both $d Q$ and $d W$ well-defined and measurable quantities. Of course, this modern definition of heat is also restricted to closed systems. A generalization to open systems will be given latter in this article.

Let us consider four basic requirements for any physically admissible definition of heat: (i) a complete distinction between open and closed systems, (ii) nonredundancy, (iii) natural variables for the thermodynamic potentials, and (iv) a sound, complete, and intuitive generalization of classical thermodynamic expressions. A detailed discussion of the requirements is as follows.

(i) By a complete distinction between open and closed systems, we mean that the definition of heat must consider the important fact that entropy $S$ and the internal energy $U$ are extensive thermodynamic quantities and both vary when the mass of the open system changes. Regarding the internal energy [5]:

"It is therefore expected that the usual version of the first law of thermodynamics for closed systems, namely $d U=d Q+d W$, will not be valid for open systems."

As Kondepudi \& Prigogine remark [1]: "For open systems, there is an additional contribution due to the flow of matter $d U_{\text {matter }}$

$d U=d Q+d W+d U_{\text {matter }}$

A similar extension is expected for the second law. Effectively, the DeDonder entropic term ${ }^{\mathbf{f}}$ for open systems is $[1,3,7]$

$d_{e} S=\frac{d Q}{T}+(d S)_{\text {matter }}$

(ii) A definition should not be devoid of distinctive physical meaning. A hypothetical definition of heat as $d Q^{\text {void }} \equiv d U$ will be rejected because it merely replaces one letter by another.

(iii) The definition of heat would use natural variables for the thermodynamic potentials instead of non-natural variables. For example, the internal energy $U$ is not a thermodynamic potential in a temperature, volume, composition, $(T, V, N)$, space.

(iv) The sound, complete, and intuitive generalization implies that the definition of heat for open thermodynamic systems should reduce to the corresponding classical expressions for closed systems in a simple and natural way.

In the next section, it will be verified that the available definitions of heat do not satisfy all the requirements, confirming the non-equivalence found by Smith [5]:

"In irreversible thermodynamics [...] there exist several definitions of heat flux [...] although many authors employ non-equivalent definitions (see e.g. deGroot and Mazur 1962)"

\section{Review of Available Definitions of Heat Flux}

Let us begin with one-component elements of volume, at rest, that can interchange internal energy and matter, without chemical reactions $\sigma_{N}=0$, and that verify the generalized Gibbs equation [3] ${ }^{\mathbf{c}}$

$T d s=d u-\mu d n$

for thermodynamic temperature $T$, density of entropy $s$, density of internal energy $u$, chemical potential $\mu$, and mole unit per volume $n$.

The entropy flux $\mathbf{J}_{S}$ is given by [4]

$\mathbf{J}_{S}=\frac{\mathbf{J}_{U}-\mu \mathbf{J}_{N}}{T}$

where $\mathbf{J}_{U}$ and $\mathbf{J}_{N}$ are the flows of internal energy and matter, respectively. This will be the starting point in the review of the different definitions of heat flux proposed up to now. More general systems will be considered in Section 5.

A first definition of heat flux is $\mathbf{J}_{Q^{D M}} \equiv \mathbf{J}_{U}$. Using this definition, Eq. (9) can be rewritten as

$\mathbf{J}_{S}=\frac{\mathbf{J}_{Q} D M-\mu \mathbf{J}_{N}}{T}$

The flux $\mathbf{J}_{Q^{D M}}$ is used by DeGroot \& Mazur [13], Fox [8], and Jou et al., $[9,10]$ in extended thermodynamics -in this case with Eq. (10) generalized to an extended thermodynamic space-; and it is the standard in the kinetic theory of diluted gases $[7,10]$. Notice this section deals with systems at rest, for moving systems with barycentric velocity $\mathbf{v}$ the above expression changes to $\mathbf{J}_{Q^{D M}}=\mathbf{J}_{U}-$ $u \mathbf{v}$.

This first definition of heat flux does not satisfy the requirements (i), (ii), and (iv) presented in the Section 2. A detailed discussion is given next.

Effectively, the flux $\mathbf{J}_{Q^{D M}}$ does not completely distinguish between open and closed systems. Substituting $\mathbf{J}_{Q D M}$ in the balance equation for the internal energy of a homogeneous mono-component thermodynamic system, at rest, without chemical reaction and in the absence of an external field, integrating over the volume $V$ of the system, and multiplying by $d t$, one obtains [7]

$d U=d Q^{D M}+d W$

Where $d W=-p d V$, with $p$ denoting pressure. This expression disagrees with the first law for open systems Eq. (6) because Eq. (11) is missing a $d U_{\text {matter }}$ term for matter exchange.

The underlying physical reason why $\mathbf{J}_{Q^{D M}}$ does not distinguish between open and closed systems, at the energetic level, is that the internal energy $U$ is an extensive thermodynamic quantity and, therefore, $\mathbf{J}_{U}=\mathbf{J}_{Q^{D M}}$ does not differentiate between changes in the internal energy due to flows of mass -transferring the internal energy per particle in the flow- and changes due to genuine heat flows.

The flux $\mathbf{J}_{Q^{D M}}$ is redundant. Any instance of $\mathbf{J}_{Q^{D M}}$ in the equations could be substituted by $\mathbf{J}_{U}$ without physical or mathematical changes. Just compare Eq. (9) and Eq. (10). At the same time, any instance of "heat flux" in the text of the above references [7-10] could be reverted to "internal energy flux" without any appreciable change. It is 
superfluous to introduce this concept of heat in the formalism. This defect is more evident when $\mathbf{J}_{Q^{D M}}$ is compared to the other definitions considered in this work, which are non-redundant and really introduce a physical concept of heat flux different from the physical concept of internal energy flux.

The formalism introduced by DeGroot \& Mazur is not backward compatible with classical thermodynamics. Indeed, they apply $\mathbf{J}_{Q^{D M}}$ to thermoelectric phenomena only after redefining internal energy -see their equation XIII.32in presence of electromagnetic fields [7]. Their redefinition is not compatible with the usual meaning of internal energy as the energy of a system at rest in absence of external fields [3] and it does not consider the Coulomb interaction energy between particles within the system, for example. As is now well-known, the van der Waals equation for the internal energy contains a term due to the interactions between molecules in a gas. Their redefinition of the wellestablished concept of internal energy is clearly motivated by their need to identity $\mathbf{J}_{Q^{D M}}$ with $\mathbf{J}_{U}$-see the corresponding redefinition of heat flux in Eq. XIII.33- [7].

There are no objective reasons for using two different concepts of internal energy at once: one in classical thermodynamics -where internal energy includes the interaction energy between particles- and another concept when using $\mathbf{J}_{Q^{D M}}$ and $d Q^{D M}$ in modern thermodynamics.

These three disadvantages of $\mathbf{J}_{Q} D M$ are a motivation for the search of improved definitions of heat flux for open systems.

A second definition of heat flux is $\mathbf{J}_{Q^{C}} \equiv \mathbf{J}_{U}-\mu \mathbf{J}_{N}$. Now the entropy flux Eq. (9) takes the simple form

$\mathbf{J}_{S}=\frac{\mathbf{J}_{Q} C}{T}$

This definition of heat is used by Callen [4]. Misner et al., [11] use a uncommon variant in curved space time thermodynamics -in this case with Eq. (9) formulated in curved spacetime.

This heat flux $\mathbf{J}_{Q}$ C does not satisfy the requirement (i) presented in the Section 2. Effectively, if one integrates Eq. (12) over the area $A$ of an isothermal system, and multiplies by $d t$ it is obtained

$d_{e} S=\frac{d Q^{C}}{T}$

which disagree with the DeDonder entropic term for open systems Eq. (7), because Eq. (13) is missing the $(d S)_{\text {matter }}$ term for matter exchange.

The underlying physical reason why $\mathbf{J}_{Q^{C}}$ does not distinguish between open and closed systems, at the entropic level, is that entropy $S$ is an extensive quantity and, therefore, Eq. (12) does not differentiate between changes in the entropy due to flow of mass -transferring the entropy per particle in the flow- and changes due to genuine heat flows.

Precisely Callen introduces his heat flux $\mathbf{J}_{Q^{C}}=T \mathbf{J}_{S}$ "in analogy" [4] with the ordinary $\delta Q=T d S$ of classical thermodynamics. The problem is that $\delta Q=T d S$ is not valid for open systems $[1,3,7],{ }^{\mathbf{f}}$ and the same physical defect is inherited by $\mathbf{J}_{Q} C=T \mathbf{J}_{S}$.

It is interesting to remark a kind of complementarity between $\mathbf{J}_{Q^{D M}}$ and $\mathbf{J}_{Q^{C}}$. If one only considers the $\mathbf{J}_{U}$ term, as
$\mathbf{J}_{Q^{D M}}$ does, one has problems with the internal energy; one the other hand, if one adds the $-\mu \mathbf{J}_{N}$ term, as $\mathbf{J}_{Q^{c}}$ does, one has problems with the entropy. A new definition of heat that considers the extensive character of both entropy and internal energy is evidently needed.

In a first step of their study of open systems, Kondepudi \& Prigogine [1] apply a change of variable, $(u, n) \rightarrow(T, n)$, to the generalized GibbsEq. (8). The ordinary entropy, internal energy, and chemical potential functions are substituted by $\hat{s}=\hat{s}(T, n), \hat{u}=\hat{u}(T, n)$, and $\hat{\mu}=\hat{\mu}(T, n)$, respectively. As a consequence, the entropy flux Eq. (9) is modified to

$\mathbf{J}_{\hat{S}}=\frac{\mathbf{J}_{\widehat{U}}-\widehat{\mu} \mathbf{J}_{N}}{T}$

In a second step, Kondepudi \& Prigogine [1] obtain the next identity

$\hat{\mu}=\hat{u}_{n}-T \hat{s}_{n}$

Above $\hat{s}_{n} \equiv(\partial \hat{s} / \partial n)_{T}$ is a partial molar entropy and $\hat{u}_{n} \equiv(\partial \hat{u} / \partial n)_{T}$ is a partial molar energy. Substituting the identity in the entropy flux Eq. (14) they finally obtain

$\mathbf{J}_{\hat{S}}=\frac{\mathbf{J}_{Q} K P}{T}+\hat{s}_{n} \mathbf{J}_{N}$

for a heat flux $\mathbf{J}_{Q^{K P}} \equiv \mathbf{J}_{\widehat{U}}-\hat{u}_{n} \mathbf{J}_{N}$. This $\mathbf{J}_{Q K P}$ is used by Kondepudi \& Prigogine [1], by Haase [3], and by Smith [5].

This third definition of heat flux does not satisfy the requirements (iii) and (iv) presented in the Section 2. A detailed discussion is given next.

It is evident that the heat flux $\mathbf{J}_{Q} K P$ cannot be defined in the thermodynamic space of natural variables. One can work in the natural energy-composition space when obtaining the entropy flux Eq. (9) from the fluxes of mass and internal energy, but one is forced to switch to a temperature-composition space if one wants to obtain the entropy flux from the heat flux and the flux of mass Eq. (16).

There are no objective reasons for introducing a change of variables before using a concept of heat flux.

The backward incompatibility with the classical expression for closed systems can be shown as follows. Using $\mathbf{J}_{Q^{K P}}$ in the balance equation for the internal energy of a homogeneous thermodynamic closed system, at rest, without chemical reactions and in absence of external field, integrating over the volume $V$ of the system, and multiplying by $d t$ one obtains

$d \widehat{U}=d Q^{K P}+d \widehat{W}$

which introduces a departure from the ordinary first law for closed systems $d U=d Q-p d V$, because $d Q^{K P} \neq T d S$ and $d \widehat{W} \neq-p d V$; see also the boxed equation 2.2.12 in [1].

It is worth to mention that Kondepudi \& Prigogine [1] redefine their heat flux $\mathbf{J}_{Q K P}$ in presence of electromagnetic fields -see the equation 15.4 .20 in [1]-. Initially they consider a definition that satisfies $\mathbf{J}_{\widehat{U}}=\mathbf{J}_{Q}{ }^{K P}+\hat{u}_{n} \mathbf{J}_{N}$; however, this changes to $\mathbf{J}_{\widehat{U}}=\mathbf{J}_{Q} K P+\hat{u}_{n}^{0} \mathbf{J}_{N}$ in presence of fields, where $\hat{u}_{n}^{0}$ is computed in absence of fields. Their more general definition implies a strange mixture of field- 
dependent and field-less terms, even in the same equations! Indeed, the above $\mathbf{J}_{\widehat{U}}$ is the flux associated to $\hat{u}_{n}$, not to $\hat{u}_{n}^{0}$. Moreover, their treatment is full of typographical errors and inconsistencies [19].

There are no objective reasons for using three different concepts of internal energy at once: the ordinary $U$ in classical thermodynamics, and both $\widehat{U}$ and $\widehat{U}^{0}$ when using $\mathbf{J}_{Q}{ } P$ in irreversible thermodynamics.

After showing that the available definitions of heat flux are redundant, do not properly describe the thermodynamics of open systems, and do not use natural variables for the thermodynamic potentials -introducing a departure from many classical thermodynamics expressions-, a new definition will be proposed.

\section{New Definition of Heat Flux}

Let us continue considering the simple system of the Section 3 for the sake of comparison with the previous definitions. More general systems will be considered in the next section.

The starting point is Eq. (9) again. A new definition of heat can be obtained by considering thermal properties in the chemical potential that were not included in the previous definitions $\mathbf{J}_{Q^{D M}}$ and $\mathbf{J}_{Q^{C}}$-recall that $\mathbf{J}_{Q^{D M}}$ ignored the $\mu \mathbf{J}_{N}$ term, whereas $\mathbf{J}_{Q} C$ included this term without any analysis-. No change of variables like that in $\mathbf{J}_{Q}$ KP is needed here. The central element will be the Euler equation associated to Eq. (8)

$T s=u+p-\mu n$

Notice that the work is continued in a thermodynamic space $(u, n)$ of natural variables, because $\mu=\mu(u, n)$, $s=s(u, n), p=p(u, n)$, and $T=T(u, n)$ in the Euler equation.

Using Eq. (18), the entropy flux Eq. (9) can be rewritten as

$\mathbf{J}_{S}=\frac{\mathbf{J}_{Q}}{T}+\left(\frac{s}{n}\right) \mathbf{J}_{N}$

with a new heat flux defined by

$\mathbf{J}_{Q} \equiv \mathbf{J}_{U}-\left(\mu+\frac{T s}{n}\right) \mathbf{J}_{N}$

This new definition of heat flux satisfies all the physical requirements (i)-(iv) presented in the section 2. A detailed discussion is given next.

It is evident that this new heat flux is non-redundant and that introduces a concept of heat flux different from the concept of internal energy flux. Moreover, the physical interpretation is intuitive. For instance, the parenthesized term in Eq. (19) is a molar entropy. ${ }^{\mathbf{i}}$ Therefore, Eq. (19) implies that $\mathbf{J}_{Q}$ is proportional to a 'thermal' entropy flow $\mathbf{J}_{S}^{*}$ where the entropy transferred through a mass flow has been subtracted

$\mathbf{J}_{Q}=T \mathbf{J}_{S}^{*} \equiv T\left[\mathbf{J}_{S}-\left(\frac{s}{n}\right) \mathbf{J}_{N}\right]$

Notice that $\mathbf{J}_{Q}=T \mathbf{J}_{S}$ only holds for closed systems, which means that that $\mathbf{J}_{Q}$ allows a physical distinction between open and closed systems at the entropic level. Effectively, integrating Eq. (19) over the area $A$ of an isothermal system, and multiplying by $d t$, one obtains the DeDonder entropic term Eq. (7) for open systems with

$$
\left(d_{e} S\right)_{\text {matter }} \equiv\left(\frac{S}{N}\right) d_{e} N
$$

The new $\mathbf{J}_{Q}$ also allows a physical distinction between open and closed systems at the energetic level because for open systems $\mathbf{J}_{U} \neq \mathbf{J}_{Q}$. This other advantage can be shown by using $\mathbf{J}_{Q}$ in the balance equation for the internal energy of a homogeneous thermodynamic system, at rest, without chemical reactions and in absence of external field; by integrating over the volume $V$ of the system and multiplying by $d t$. The final result is the first law of thermodynamics for open systems Eq. (6) with

$d U_{\text {matter }} \equiv\left(\mu+\frac{T S}{N}\right) d_{e} N$

This new heat flux $\mathbf{J}_{Q}$ is able to distinguish between open and closed systems completely, because $\mathbf{J}_{Q}$ considers the extensive thermodynamic character of both the entropy $S$ and the internal energy $U$. Effectively, Eq. (19) distinguishes changes in the entropy due to flows of mass from changes due to genuine heat flows, whereas $\mathbf{J}_{U}=$ $\mathbf{J}_{Q}+(\mu+T s / n) \mathbf{J}_{N}$ distinguishes between changes in the internal energy due to genuine heat flows and those due to flows of mass. Notice that for simple closed systems $\mathbf{J}_{Q}=\mathbf{J}_{Q^{D M}}=\mathbf{J}_{Q^{C}}$.

Moreover, $\mathbf{J}_{Q}$ is defined in a thermodynamics space of natural variables and allows for a smooth generalization of classical thermodynamic expressions to open systems. For closed systems $\left(d_{e} S\right)_{\text {matter }}=d U_{\text {matter }}=0$ and the ordinary first law for closed systems $d U=d Q-p d V$ is recovered because $d W=-p d V$ and $d Q=T d S$.

In the following section, it will be presented the new general definition of heat and its further advantages.

\section{Heat for General Thermodynamic Systems}

Instead considering a simple system as we did in the Section 3, it will be considered now a generic thermodynamic system. The analysis will start with a multicomponent element of volume that can interchange internal energy, matter, and a collection of "work coordinates" [3] whose densities are $z_{j}-$. This thermodynamic element of volume verifies a generalized Gibbs equation [3] ${ }^{\mathbf{c}}$

$T d s=d u-\sum_{j} \zeta_{j} d z_{j}-\sum_{k} \mu_{k} d n_{k}$,

for thermodynamic temperature $T$, density of entropy $S$, density of internal energy $u$, and chemical potential $\mu_{k}$ and mole unit per unit volume $n_{k}$ of component $k$. The coefficients $\zeta_{j}$ are "work coefficients" [3]. Examples of work coefficients and work coordinates are given in the literature for specific thermodynamic interactions, such as those due to transfer of density of charge $q$ across a potential difference $\phi$, change of density of electric dipole moment $\mathbf{p}$ in presence of an electric field $\mathbf{E}$, and change of density of magnetic dipole moment $\mathbf{m}$ in presence of a magnetic field $\mathbf{B}[1,3]$

$$
\sum_{j} \zeta_{j} d z_{j}=\phi d q-\mathbf{E} d \mathbf{p}-\mathbf{B} d \mathbf{m}+\cdots
$$


The balance equation for entropy has the same form as Eq. (3), with a generalized density of production of entropy

$\sigma_{S}=\frac{\sigma_{U}-\sum_{j} \zeta_{j} \sigma_{Z_{j}}-\sum_{k} \mu_{k} \sigma_{N_{k}}}{T}+\mathbf{J}_{U} \cdot \nabla\left(\frac{1}{T}\right)-\sum_{j} \mathbf{J}_{Z_{j}} \cdot \nabla\left(\frac{\zeta_{j}}{T}\right)-$

$\sum_{k} \mathbf{J}_{N_{k}} \cdot \nabla\left(\frac{\mu_{k}}{T}\right)$

and with a generalized entropy flux

$\mathbf{J}_{S}=\frac{\mathbf{J}_{U}-\sum_{j} \zeta_{j} \mathbf{J}_{z_{j}}-\sum_{k} \mu_{k} \mathbf{J}_{N_{k}}}{T}$

In the above expressions, $\mathbf{J}_{U}, \mathbf{J}_{Z_{j}}$, and $\mathbf{J}_{N_{k}}$ are the flows of internal energy, work coordinate $j$, and component $k$, respectively; whereas $\sigma_{U}, \sigma_{Z_{j}}$, and $\sigma_{N_{k}}$ are their corresponding densities of production. For instance, the density of production of component $k$ by chemical reactions can be expressed in terms of the reactions velocities $v_{\alpha}$ and the corresponding stoichometric coefficients $v_{\alpha k}$ as $\sigma_{N_{k}} \equiv \sum_{\alpha} v_{\alpha} v_{\alpha k}$.

The starting point of the analysis is Eq. (27). Once again, the new definition of heat for general systems can be obtained by considering thermal properties in the chemical potential. The generalized Euler equation associated to Eq. (24) is

$T s=u+p-\sum_{j} \zeta_{j} z_{j}-\sum_{k} \mu_{k} n_{k}$

Notice that it is defined in a thermodynamic space $\left(u,\left\{z_{k}\right\},\left\{n_{k}\right\}\right)$ of natural variables.

A problem, which was absent in the one-component system, appears now. The chemical potentials are interrelated and the thermal properties of the chemical potential of component $k$ cannot be isolated in the generalized Euler equation. This difficulty can be solved if one uses Eq. (28) to obtain the next pseudo-identity

$\frac{1}{R}\left(T s-u-p+\sum_{j} \zeta_{j} z_{j}\right)=-\mu_{k} n_{k}$

With $R \equiv \sum_{k}$ the total number of components. This pseudo-identity will be the central element for the definition of heat flux for general systems. Using Eq. (29), the density of production of entropy Eq. (26) can be rewritten as

$\sigma_{S}=\frac{\sigma_{U}-\sum_{j} \zeta_{j} \sigma_{Z_{j}}-\sum_{k} \mu_{k} \sigma_{N_{k}}}{T}+\mathbf{J}_{Q} \cdot \nabla\left(\frac{1}{T}\right)-\sum_{j} \mathbf{J}_{Z_{j}} \cdot\left(\frac{\nabla \zeta_{j}}{T}\right)-$

$\sum_{k} \mathbf{J}_{N_{k}} \cdot \nabla\left(\frac{\psi_{k}}{T}\right)$

and the entropy flux Eq. (27) rewritten as

$\mathbf{J}_{S}=\frac{\mathbf{J}_{Q}}{T}+\sum_{k}\left(\frac{s}{R n_{k}}\right) \mathbf{J}_{N_{k}}$

with $\psi_{k} \equiv\left(1 / R n_{k}\right) \nabla \mathrm{p}-\sum_{j}\left(z_{j} / R n_{k}\right) \nabla \zeta_{j}$ and a generalized heat flux

$\mathbf{J}_{Q} \equiv \mathbf{J}_{U}-\sum_{j} \zeta_{j} \mathbf{J}_{Z_{j}}-\sum_{k}\left(\mu_{k}+\frac{T s}{R n_{k}}\right) \mathbf{J}_{N_{k}}$

This extension to general systems of the new definition of heat flux continues satisfying all the physical requirements (i)-(iv) presented in the Section 2.

Notice that the factor $\left(s / R n_{k}\right)$ has units of molar entropy, which implies that $\left(\mu_{k}+T s / R n_{k}\right)$ has units of molar energy. The new heat flux $\mathbf{J}_{Q}$ for general systems continues taking into account the extensive thermodynamic character of both the entropy $S$ and the internal energy $U$. This allows a direct extension of the analysis for simple systems -presented in the Section 4- to multi-component generalized thermodynamic systems. Effectively, Eq. (31) implies that $\mathbf{J}_{Q}$ is proportional to a 'thermal' entropy flow $\mathbf{J}_{S}^{*}$ where the entropy transferred through the mass flows has been substracted

$\mathbf{J}_{Q}=T \mathbf{J}_{S}^{*} \equiv\left[\mathbf{J}_{S}-\sum_{k}\left(\frac{s}{R n_{k}}\right) \mathbf{J}_{N_{k}}\right]$

The new definition Eq. (32) is applicable to generalized thermodynamic systems associated to the Euler Eq. (28). A particular case is thermal radiation for which $\mathbf{J}_{Q}=\mathbf{J}_{U}=$ $\mathbf{J}_{Q^{D M}}=\mathbf{J}_{Q^{C}}$. This result is natural because the new definition of heat has been obtained by considering thermal properties in the chemical potential and for photons $\mu=0$.

The corresponding generalizations of the DeDonder entropic term Eq. (22) and of the $d U_{\text {matter }}$ in Eq. (23) are easy to obtain.

Integrating the definition Eq. (32) of the new heat flux $\mathbf{J}_{Q}$ over the area $A$ of an initially homogeneous system, multiplying by the time interval $d t$ needed to achieve a final equilbrium state -from the initial equilibrium state-, downgrading from modern to classical thermodynamic space, ${ }^{\mathbf{e}}$ and considering the relation between the total energy and the internal energy, one obtains a generalization of the classical Bryan \& Born definition of heat Eq. (2) to open systems

$\delta Q \equiv d E-\delta W-\sum_{k}\left(\frac{T S}{R N_{k}}\right) \delta_{e} N_{k}$

Evidently Eq. (34) reduces to Eq. (2) for closed systems. Using Eq. (31), one can also obtain the proper generalization of the classical Clausius theorem to open systems

$d S \geq \frac{\delta Q}{T}+\sum_{k}\left(\frac{S}{R N_{k}}\right) \delta_{e} N_{k}$

This work only considers a macroscopic treatment of thermodynamic systems. This treatment is not applicable, for instance, to the transition from diffusive to ballistic heat, of much interest in nanosystems or to rarefied gases for which an extended thermodynamic formalism is needed. However, at the time of writing this, there is not consensus on which is the best way to extend thermodynamics; different competing formalisms -EIT, rational, Keizer, GENERIC [8,9]- have been proposed. This issue will be investigated in a future work.

As noticed in Section 3, the macroscopic DeGroot \& Mazur flux $\mathbf{J}_{Q^{D M}}$ follows from the ordinary microscopic definition of heat flux in the kinetic theory of gases. Besides the macroscopic deficiencies reported in the Section 3, there are additional difficulties in the usual kinetic definition as the velocity average of the kinetic 'operator' $m \mathbf{c} c^{2} / 2$, where the $m$ is the particle mass and $\mathbf{c}$ the velocity of the particle with respect to the average. If the standard definition is used in the dissipation-fluctuation theorem, this does not yield the correct result for the thermal conductivity of ideal gases. The correct result is obtained when the usual kinetic 'operator' is amended with 
a $h \mathbf{c}$ term, where $h$ is the enthalpy per particle. It is particularly interesting that the correction term -see Eq. (20) - to the macroscopic DeGroot \& Mazur flux is precisely the enthalpy per mol. This shows that having a good definition of heat flux is not only of interest for the flux itself, but also for the analysis of the fluctuations of the heat flux.

\section{Conclusions}

The flux $\mathbf{J}_{Q} \equiv \mathbf{J}_{U}-\sum_{j} \zeta_{j} \mathbf{J}_{Z_{j}}-\sum_{k}\left(\mu_{k}+T s / R n_{k}\right) \mathbf{J}_{N_{k}}$ provides (i) a complete distinction between open and closed systems, (ii) non-redundancy, (iii) natural variables for the thermodynamic potentials, and (iv) a sound, complete, and intuitive generalization of classical thermodynamic expressions - such as Clausius $T d S \geq \delta Q$ and the Bryan \& Born definition Eq. (2)- to open systems.

\section{Acknowledgements:}

I wish to thank Christopher Game for calling my attention to Bryan's definition of heat and for his invaluable assistance in the preparation of this manuscript. I also wish to thank two anonymous referees for their improvements of the original manuscript.

\begin{tabular}{|c|c|}
\hline \multicolumn{2}{|c|}{ Nomenclature } \\
\hline B & magnetic field, $\mathrm{T}$. \\
\hline$\delta Q$ & heat (classical thermodynamics), J. \\
\hline$d Q$ & heat (modern thermodynamics), ${ }^{\mathbf{e}} \mathrm{J}$. \\
\hline$\delta W$ & total work, $\mathrm{J}$. \\
\hline$d W$ & total work (modern thermodynamics), ${ }^{\mathbf{e}} \mathrm{J}$. \\
\hline$\delta W_{T D}$ & thermodynamic work, J. \\
\hline$\delta_{e} N_{k}$ & $\begin{array}{l}\text { change in composition due to exchanges with } \\
\text { exterior, mol. }\end{array}$ \\
\hline EIT & Extended Irreversible Thermodynamics. \\
\hline $\mathbf{E}$ & electric field, $\mathrm{V} \cdot \mathrm{m}^{-1}$. \\
\hline GENERIC & $\begin{array}{l}\text { General Equation for the Non-Equilibrium } \\
\text { Reversible-Irreversible Coupling }\end{array}$ \\
\hline $\mathbf{J}_{N}$ & matter flux, $\mathrm{mol} \cdot \mathrm{s}^{-1} \cdot \mathrm{m}^{-2}$ \\
\hline $\mathbf{J}_{Q}$ & heat flux, $\mathrm{J} \cdot \mathrm{s}^{-1} \cdot \mathrm{m}^{-2}$. \\
\hline $\mathbf{J}_{S}$ & entropy flux, $\mathrm{J} \cdot \mathrm{K}^{-1} \cdot \mathrm{s}^{-1} \cdot \mathrm{m}^{-2}$. \\
\hline $\mathbf{J}_{S}^{*}$ & thermal entropy flux, $\mathrm{J} \cdot \mathrm{K}^{-1} \cdot \mathrm{s}^{-1} \cdot \mathrm{m}^{-2}$. \\
\hline $\mathbf{J}_{U}$ & internal energy flux, $\mathrm{J} \cdot \mathrm{s}^{-1} \cdot \mathrm{m}^{-2}$. \\
\hline$\mu$ & chemical potential, $\mathrm{J} \cdot \mathrm{mol}^{-1}$. \\
\hline $\mathbf{m}$ & density of magnetic dipole moment, $\mathrm{A} \cdot \mathrm{m}^{-1}$. \\
\hline$v_{\alpha k}$ & stoichiometric coefficient, adimensional. \\
\hline$n$ & mole unit per unit volume, $\mathrm{mol} \cdot \mathrm{m}^{-3}$. \\
\hline$N$ & composition, mol. \\
\hline$\phi$ & electric potential difference, $\mathrm{V}$. \\
\hline$p$ & pressure, $\mathrm{Pa}$ \\
\hline p & density of electric dipole moment, $\mathrm{C} \cdot \mathrm{m}^{-2}$. \\
\hline$q$ & density of charge, $\mathrm{C} \cdot \mathrm{m}^{-3}$. \\
\hline$R$ & $\begin{array}{l}\text { total number of chemical components, } \\
\text { adimensional. }\end{array}$ \\
\hline$\sigma_{N}$ & chemical production, $\mathrm{mol} \cdot \mathrm{s}^{-1} \cdot \mathrm{m}^{-3}$. \\
\hline$s$ & density of entropy, $\mathrm{J} \cdot \mathrm{K}^{-1} \cdot \mathrm{m}^{-3}$. \\
\hline$\hat{s}_{n}$ & partial molar entropy, $\mathrm{J} \cdot \mathrm{K}^{-1} \cdot \mathrm{mol}^{-1}$ \\
\hline$t$ & time, s. \\
\hline$T$ & thermodynamic temperature, $\mathrm{K}$. \\
\hline$u$ & density of internal energy, $\mathrm{J} \cdot \mathrm{K}^{-1} \cdot \mathrm{m}^{-3}$. \\
\hline$\hat{u}_{n}$ & partial molar internal energy, $\mathrm{J} \cdot \mathrm{mol}^{-1}$. \\
\hline$\hat{u}_{n}^{0}$ & $\begin{array}{l}\text { partial molar internal energy in absence of } \\
\text { fields, } \mathrm{J} \cdot \mathrm{mol}^{-1} \text {. }\end{array}$ \\
\hline$U$ & internal energy, $\mathrm{J}$. \\
\hline$v_{\alpha}$ & reaction velocity, $\mathrm{mol} \cdot \mathrm{m}^{3} \cdot \mathrm{s}^{-1}$. \\
\hline
\end{tabular}

volume, $\mathrm{m}^{3}$. generalized work coefficient, variable units. density of generalized work coordinate, variable units.

\section{Notes:}

[a] IUPAC recommendation for the signs -work is considered positive if increases the energy of the system- is used in this article as in most of the literature. See, for instance, [1, 3, 4, 5, 9].

[b] Callen uses the old term "closed" instead of the more modern and adequate term "isolated" for referring to systems that cannot interchange energy or matter with the surrounds $[1,3]$.

[c] This is an excellent approximation for systems where there are not large gradients and/or fast processes. When the local approximation does not hold, the formalism of extended thermodynamics is needed. See [9] and references cited therein.

[d] The term "flux" is used here for $\mathbf{J}_{Y}$ because readers must be more familiar with this old terminology. However, recent official recommendation of IUPAP and IUPAC is the term "flux density" for $\mathbf{J}_{Y}$ [6]. Some references are completely inconsistent regarding the terminology. For instance, DeGroot \& Mazur consider that $\mathbf{J}_{e}$ is both the "energy flux" and the "energy flux per unit surface and unit time" [7]. Callen initially defines "flux" in agreement with recent IUPAP/IUPAC terminology, but latter redefines "flux" as the $x, y$, or $z$ component of a "current density" [4], ignoring the different units. Kondepudi \& Prigogine [1] offer similar inconsistencies.

[e] Classical thermodynamics is defined in a timeless thermodynamic space associated to "idealized, infinitely slow, reversible processes" [1]. This forces the use of imperfect differentials $\delta$, for quantities such as heat and work. Modern thermodynamics is defined in an extended thermodynamic space without such idealizations, which "avoids the use of imperfect differentials" [1].

[f] The DeDonder equation $d_{e} S=d Q / T$, associated with modern thermodynamics, should not be confused with the Clausius equation $d S=\delta Q / T$ of classical thermodynamics. In the first place, the DeDonder equation is for $d_{e} S$, whereas the Clausius equation is for $d S$. In general, $d_{e} S \neq d S$, which implies that the Clausius equation is only valid forreversible processes, whereas the DeDonder equation is valid for irreversible processesas well. In the second place, $d Q$ in the DeDonder equation uses perfect differentials, whereas the Clausius equation relies on imperfect differentials such as $\delta Q$. See previous Note for details.

[g] Misner, Thorne, \& Wheeler [11] consider only simple thermodynamic systems and absence of chemical reactions. They begin by introducing a "heat-flux 4vector" $\mathbf{q}$ with components $\left(0, q^{1}, q^{2}, q^{3}\right)$ defined in the rest-frame of the element of volume; they vaguely define the spatial components as "energy per unit time crossing unit surface" -see their 22.16.b-, without specifying what energy. They define an "entropy 4vector" as $\mathbf{s} \equiv s \mathbf{u}+\mathbf{q} / T$-their 22.16.e- and postulate the "second law of thermodynamics" in the form $\nabla^{4} \cdot \mathbf{s} \geq 0$-their 22.16.g-, with $\nabla^{4}$ the four-divergence. This is all strange and incorrect. In the first place, they do not unambiguously define what is the energy 
associated to their heat-flux. In the second place, their 4-vector $\mathbf{s}$ cannot be an entropy because it has units of entropy per unit area and per unit time; $\mathbf{s}$ is, in reality, an entropy-flux 4-vector. ${ }^{\mathbf{d}}$ In the third place, the authors mix local and material flows when define $\mathbf{s}$ as their heat-flux 4-vector plus an 'convective' term. As a consequence, the internal energy flux 3-vector associated to the entropy flux 3 -vector is measured locally, at a given spacetime point, whereas the heatflux 3-vector associated to their four-vector $\mathbf{q}$ cannot be measured locally. Compare this with Callen and his use of a local heat-flux 3-vector, which is locally measured [4].

[h] A detailed analysis of their work reveals that 15.4.20 and 15.4.23, defining the heat flux and the source of heat respectively, contain $u_{k}$ instead $u_{k}^{0}$; both definitions contain dummy $\sum_{i}$ symbols that have to be eliminated. The authors give 15.5.12 in terms of an "electric current" I which they define in the text; however, when restating the density of production of entropy -A15.1.13- in terms of their $\mathbf{J}_{Q^{K P}}$ they introduce a $\mathbf{I}_{k}$ which they define nowhere -one can guess that $\mathbf{I}_{k}$ represents the contribution of ion $k$ to the "electric current"-. They use everywhere $-\nabla \psi$ for the electric field, except in table 15.1 where they use $-\nabla \phi$. Several expressions in the same chapter contain typos such as $\partial / d t$. Their 15.5 .11 is dimensionally incorrect, but following the text one can guess that $\mathbf{J}_{k}^{\psi}$ substituted $\mathbf{J}_{U}$. In section 16.3 they start using $\mathbf{I}_{e}$ for "electric current", but after nine equations suddenly change to the older notation I, which then rename as "current density". A $T$ is missing in 10.1 .15 for electrical conduction. Both the figure 10.3 and the equation 10.1.11 confound chemical and electrochemical potentials; etcetera.

[i] Molar quantities are defined as $Y / N$. IUPAC recommends the notation $Y_{m}$ for molar quantities. Notice that $Y / N=y / n$.

\section{References:}

[1] D. K. Kondepudi and I. Prigogine, Modern Thermodynamics. Chichester: John Wiley \& Sons, 1998.

[2] G. H. Bryan, Thermodynamics, an introductory treatise dealing mainly with first principles and their direct applications. Leipzig: B. G. Teubner, 1907.

[3] R. Haase, "Survey of fundamental laws," in Physical Chemistry, An Advanced Treatise, Volume I Thermodynamics, W. Jost, Ed. London: Academic Press, 1971.

[4] H. B. Callen, Thermodynamics and an Introduction to Thermostatistics, 2nd ed. New York: John Wiley \& Sons, 1985.

[5] D. A. Smith, "Definition of Heat in Open Systems," Aust. J. Phys., vol. 33, pp. 95-105,.1990.

[6] E. R. Cohen, Ed. Quantities, Units and Symbols in Physical Chemistry, 2nd Printing. Cambridge: IUPAC \& RSC Publishing, 2008.

[7] S. R. De Groot and P. Mazur, Non-equilibrium thermodynamics. New York: Courier Dover Publications, 1984.

[8] R. F. Fox, "Non-equilibrium thermodynamics," in Encyclopedia of Chemical Physics and Physical Chemistry, Volume I Fundamentals, J. H. Moore and N. D. Spencer, Eds. Bristol: IOP Publishing Bristol, 2001.

[9] D. Jou, J. Casas-Vázquez, and G. Lebon, Extended Irreversible Thermodynamics, 4th Ed. New York: Springer, 2010.

[10] R. Balescu, Statistical dynamics; matter out of equilibrium. London: Imperial College Press, 1997.

[11] C. Misner, K. S. Thorne, and J. A. Wheeler, Gravitation. San Francisco: W. H. Freeman \& Co., 1973. 